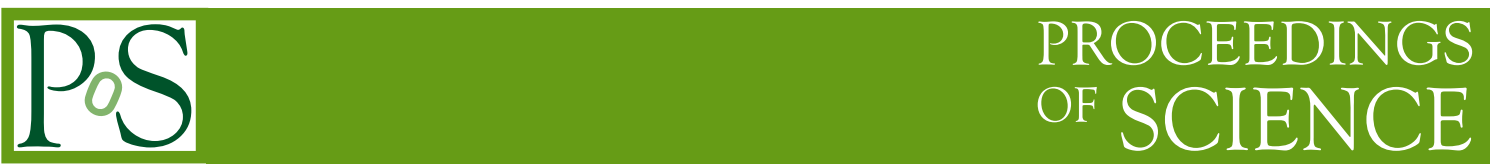

\title{
Neutrino masses and cosmology
}

\author{
Sergio Pastor ${ }^{* \dagger}$ \\ Instituto de Física Corpuscular (CSIC-Universitat de València) \\ Ed. Institutos de Investigación, Apdo. 22085, E-46071 Valencia, Spain \\ E-mail: pastor@ific.uv.es
}

Cosmology can provide information on the absolute scale of neutrino masses, complementary to the results of tritium beta decay and neutrinoless double beta decay experiments. We show how the analysis of data on cosmological observables, such as the anisotropies of the cosmic microwave background or the distribution of large-scale structure, combined with other experimental results, provides an upper bound on the sum of neutrino masses. We also discuss how future cosmological experiments are expected to be sensitive to neutrino masses well into the sub-eV range.

International Europhysics Conference on High Energy Physics

July 21st - 27th 2005

Lisboa, Portugal

\footnotetext{
*Speaker.

${ }^{\dagger}$ Work supported by the Spanish grants BFM2002-00345 and GV/05/017 of Generalitat Valenciana, as well as by a Ramón y Cajal contract of MEC.
} 


\section{Massive neutrinos as dark matter}

Neutrinos are very abundant in the Universe, in number only slightly smaller than that of relic photons. After being created in earlier epochs, relic neutrinos influence various cosmological stages, playing an important role that has been used to derive bounds on non-standard neutrino properties, alternative to the limits from terrestrial neutrino experiments (see e.g. [1] for an extensive review on many aspects of neutrino cosmology). The idea of neutrinos as dark matter (DM) particles has been widely discussed since the 1970s, since there are two facts in their favour: they definitely exist and $\mathrm{eV}$ neutrino masses produce a contribution of order unity to the present energy density of the Universe, which in terms of its critical value $\rho_{c}$ is $\Omega_{v}=\rho_{v} / \rho_{c}=M_{v} /\left(93.2 h^{2} \mathrm{eV}\right)$ (where $h \equiv H_{0} /\left(100 \mathrm{~km} \mathrm{~s}^{-1} \mathrm{Mpc}^{-1}\right.$ ) is the present value of the Hubble parameter and $M_{v} \equiv \sum_{i} m_{v_{i}}$ is the total neutrino mass). Thus one easily finds an upper limit on the masses (some tens of eV) by imposing the very conservative bound $\Omega_{v}<1$.

The background of relic massive neutrinos affects the evolution of cosmological perturbations in a particular way: it erases the density contrasts on wavelengths smaller than a mass-dependent free-streaming scale. This damping of the density fluctuations on small scales is characteristic of hot dark matter (HDM) particles. In a Universe dominated by HDM, large objects such as superclusters of galaxies form first, while smaller structures like clusters and galaxies form via a fragmentation process (a top-down scenario). However, within the presently favoured $\Lambda \mathrm{CDM}$ model, dominated at late times by dark energy and where the main matter component is pressureless, there is no need for a significant contribution of HDM. Therefore, from an analysis of the available cosmological data one can find how large the neutrino contribution can be and translate it into an upper bound on the values of the neutrino masses. This bound is important because presently we have experimental evidences of flavour neutrino oscillations, which are sensitive to the squared mass differences between the three neutrino mass states $m_{1,2,3}$ : allowed ranges are $\left|\Delta m_{31}^{2}\right|=\left(2.2_{-0.8}^{+1.1}\right) \times 10^{-3}$ $\mathrm{eV}^{2}$ and $\Delta m_{21}^{2}=\left(8.1_{-0.9}^{+1.0}\right) \times 10^{-5} \mathrm{eV}^{2}$ (see e.g. [2] and references therein), which lead to two possible neutrino mass schemes, depending on the sign of $\Delta m_{31}^{2}$. For very small values of the lightest neutrino mass, the mass states follow a hierarchical scenario with minimal values of the sum $M_{v} \simeq 0.05(0.1) \mathrm{eV}$ in the normal (inverted) case, while for masses much larger than the differences all neutrinos share in practice the same mass $m_{0} \simeq M_{v} / 3$ (mass degenerate region).

Cosmology is at first order sensitive to the total mass if all neutrino states have the same number density, providing information on $m_{0}$ but blind to neutrino mixing angles or possible $\mathrm{CP}$ violating phases. Instead, tritium decay and neutrinoless double beta decay [4] experiments are sensitive to $\sum_{i}\left|U_{e i}\right|^{2} m_{i}^{2}$ and $m_{e e} \equiv\left|\sum_{i} U_{e i}^{2} m_{i}\right|$, respectively ( $U$ is the $3 \times 3$ neutrino mixing matrix). Presently, from tritium beta decay one finds $m_{0}<2.2 \mathrm{eV}$ (95\% CL), a bound expected to be improved by the KATRIN project to reach $0.3-0.35 \mathrm{eV}$ [5]. We also have results on $m_{e e}$ from neutrinoless double beta decay experiments, which give upper bounds in the range $0.3-1.6 \mathrm{eV}$ and a claim of positive evidence for $m_{e e}[6]$. However, these results suffer from the uncertainties in the calculations of the corresponding nuclear matrix elements.

\section{Current cosmological bounds on neutrino masses}

For neutrino masses of order $\mathrm{eV}$, the free-streaming effect can be detectable in the linear matter power spectrum, reconstructed from galaxy redshift surveys. Massive neutrinos have also a smaller 


\begin{tabular}{ccl}
\hline Ref. & Bound (eV) & Data (in addition to WMAP) \\
\hline PRD 71 (2005) 043001 & 2.0 & - \\
PRD 69 (2004) 103501 & 1.7 & SDSS-gal \\
astro-ph/0507583 & 1.2 & other CMB, 2dF-gal \\
PRD 69 (2004) 123007 & 1.0 & ACBAR, 2dF-gal, SDSS-gal \\
JCAP 05 (2003) 004 & 1.0 & other CMB, (pre-WMAP), 2dF-gal, HST, SNIa \\
MNRAS 353 (2004) 747 & 0.96 & VSA, 2dF-gal \\
PLB 595 (2004) 55 & 0.75 & other CMB (pre-WMAP), 2dF-gal, SDSS-gal, HST \\
APJS 148 (2003) 175 & 0.68 & ACBAR, CBI, 2dF-gal, 2dF-bias, Ly $\alpha$ \\
astro-ph/0507503 & 0.48 & other CMB, 2dF-gal, SDSS-gal, bias \\
PRD 70 (2005) 113003 & 0.47 & other CMB, 2dF-gal, HST, SNIa, Ly $\alpha$ \\
PRD 71 (2005) 103515 & 0.42 & SDSS-gal, SNIa, SDSS-bias, Ly $\alpha$ \\
\hline
\end{tabular}

Table 1: Upper bounds on the total neutrino mass $M_{v}(\mathrm{eV}, 95 \% \mathrm{CL})$ from recent analyses of different sets of cosmological data. See the text for details.

background effect: different values of the neutrino density fraction $\Omega_{v}$ have to be compensated by small changes to the other components, modifying some characteristic times and scales in the history of the Universe, like the time of equality between matter and radiation, or the size of the Hubble radius at photon decoupling. Although neutrino masses influence only slightly the spectrum of the anisotropies of Cosmic Microwave Background (CMB) radiation, it is crucial to combine $\mathrm{CMB}$ and large-scale structure (LSS) observations, as well as other cosmological observations, in order to measure the neutrino mass, because $\mathrm{CMB}$ data give independent constraints on the cosmological parameters, and partially removes the parameter degeneracies.

We show in Table 1 a summary of recent results from analyses of cosmological data, which emphasizes the fact that a single cosmological bound on neutrino masses does not exist. Assuming that the relic neutrinos are standard, the limits depend on the underlying model (the set of cosmological parameters) and the cosmological data used. The data include results from CMB experiments (WMAP, ACBAR, DASI, VSA, CBI, Boomerang and others) and different LSS data: the shape of the matter power spectrum on large scales as found from galaxy-galaxy correlation data from galaxy redshift surveys such as 2dFGRS or SDSS (referred as 2dF/SDSS-gal in the table), the bias (normalization of the matter power spectrum) or the matter power spectrum on small scales inferred from the Lyman- $\alpha$ forest. In addition, other cosmological data can be incorporated via priors on parameters such as $h$ (HST) or $\Omega_{m}$ (SNIa data). For details, see the discussion in [7].

These results in Table 1 tell us that current cosmological data probe the region of neutrino masses where the 3 neutrino states are degenerate, with a mass $M_{v} / 3$. This mass region is conservatively bounded to values below approximately $1 \mathrm{eV}$ from CMB results combined only with galaxy clustering data from $2 \mathrm{dF}$ and/or SDSS (unnormalized matter power spectrum). The addition of further data leads to an improvement of the bounds, which reach the lowest values $0.42-0.68 \mathrm{eV}$ when data from Lyman- $\alpha$ are included or the bias is fixed.

Note that these impressive cosmological bounds on neutrino masses may change if additional cosmological parameters, beyond those included in the minimal $\Lambda \mathrm{CDM}$, are allowed. This could be the case whenever a new parameter degeneracy with the neutrino masses arises. This is the case when the radiation content of the Universe (parametrized via the effective number of neutrinos $N_{\text {eff }}$ ) 
is enhanced: the extra radiation partially compensates the effect of neutrino masses, leading to a less stringent bound on $M_{v}$ [7, 8] (see e.g. Fig. 1 in [7]). For instance, when one considers a model with four instead of three species of massive neutrinos (as suggested by the LSND experiment, where a fourth sterile neutrino is required with mass of $\mathscr{O}(\mathrm{eV})$ [2]), the upper bound found from CMB and 2dF/SDSS-gal is relaxed from 0.8 to $1.2 \mathrm{eV}$ when one of the states is much heavier than the others. Recently a degeneracy between neutrino masses and the parameter $w$, that characterizes the equation of state of the dark energy component $X\left(P_{X}=w \rho_{X}\right)$, was studied in [9]. For values $w<-1$, in the so-called phantom energy regime, it was shown that the neutrino mass bound can be relaxed to $M_{v}<1.48 \mathrm{eV}$ (95\% C.L.), compared with $M_{v}<0.65 \mathrm{eV}$ for $w=-1$.

\section{Future sensitivities to neutrino masses from cosmological observations}

Future CMB data from WMAP and Planck, combined with LSS data from larger galaxy surveys will enhance the cosmological sensitivity to neutrino masses. The pioneering calculation in ref. [10] found that the combination of Planck and SDSS data will push the bound on $M_{v}$ to approximately $0.3 \mathrm{eV}$ (see also [11]). An updated forecast analysis gave $0.21 \mathrm{eV}$ for Planck+SDSS, that could be improved to $0.13 \mathrm{eV}$ with future data from CMBpol (a very preliminary project of a future CMB satellite with better sensitivity to $\mathrm{CMB}$ polarization). Other cosmological probes of neutrino masses could reach better sensitivities in the next future. In particular one can probe the mass distribution of the Universe using either the weak gravitational lensing of background galaxies by intervening matter [12] or the distortions of CMB temperature and polarization spectra caused by gravitational lensing [13]. These two methods, alone or combined [14], are potentially sensitive to masses $0.05-0.1 \mathrm{eV}$, i.e. possibly down to the minimum values expected in the hierarchical neutrino schemes, in particular in the inverted case.

\section{References}

[1] A.D. Dolgov, Phys. Rep. 370 (2002) 333.

[2] M. Maltoni et al, New J. Phys. 6 (2004) 122; M. Maltoni, in these proceedings.

[3] J. Lesgourgues, S. Pastor and L. Perotto, Phys. Rev. D 70 (2004) 045016.

[4] Contributions of L. Vala, P. Gorla, K.T. Knöpfle and D. Muenstermann, in these proceedings. For a review, see e.g. S.R. Elliott and P. Vogel, Ann. Rev. Nucl. Part. Sci. 52 (2002) 115.

[5] K. Valerius, in these proceedings (more information at http://www-ik.fzk.de/katrin/)

[6] H.V. Klapdor-Kleingrothaus et al, Phys. Lett. B 586 (2004) 198.

[7] P. Crotty, J. Lesgourgues and S. Pastor, Phys. Rev. D 69 (2004) 123007.

[8] S. Hannestad and G. Raffelt, JCAP 0404 (2004) 008.

[9] S. Hannestad, Phys. Rev. Lett. , in press [astro-ph/0505551].

[10] W. Hu, D.J. Eisenstein and M. Tegmark, Phys. Rev. Lett. 80 (1998) 5255.

[11] S. Hannestad, Phys. Rev. D 67 (2003) 085017.

[12] K.N. Abazajian and S. Dodelson, Phys. Rev. Lett. 91 (2003) 041301.

[13] M. Kaplinghat et al, Phys. Rev. Lett. 91 (2003) 241301; J. Lesgourgues et al, astro-ph/0511735.

[14] Y.S. Song and L. Knox, Phys. Rev. D 70 (2004) 063510. 